\title{
Continuation versus discontinuation of first-line chemotherapy in patients with metastatic squamous-cell oesophageal cancer: A randomised phase II trial (E-DIS)
}

Running Head: Chemotherapy in squamous-cell oesophageal cancer

Adenis $\mathrm{A}^{\mathrm{a}, \mathrm{b}, \mathrm{c}}$, Bennouna $\mathrm{J}^{\mathrm{d}}$, Etienne $\mathrm{PL}^{\mathrm{e}}$, Bogart $\mathrm{E}^{\mathrm{f}}$, Francois $\mathrm{E}^{\mathrm{g}}$, Galais $\mathrm{MP}{ }^{\mathrm{h}}$, Ben

Abdelghani $\mathrm{M}^{\mathrm{i}}$, Michel $\mathrm{P}^{\mathrm{j}}$, Metges $\mathrm{JP}^{\mathrm{k}}$, Dahan $\mathrm{L}^{\prime}$, Conroy $\mathrm{T}^{\mathrm{m}}$, Ghiringhelli $\mathrm{F}^{\mathrm{n}}$, Drouillard $\mathrm{A}^{\circ}$,

El Hajbi $F^{a}$, Samalin $E^{b}$, Hiret $S^{d}$, Delaine-Clisant $S^{b, f}$, Mariette $C^{\# c, p}$, Penel $N^{a, f}$,

Piessen $\mathrm{G}^{\mathrm{c}, \mathrm{p}}$, Le Deley MC ${ }^{\mathrm{f}, \mathrm{q}}$

a. Département d'Oncologie Médicale, Centre Oscar Lambret, Lille, France

b. Département d'Oncologie Médicale, Institut Régional du Cancer de Montpellier, Montpellier, France

c. FREnch EsoGastric Tumor (FREGAT) working group, Lille, France

d. Département d'Oncologie Médicale, Institut de Cancérologie de l'Ouest, SaintHerblain, France

e. Centre Armoricain Radiothérapie Imagerie Oncologie, Hôpital Privé des Côtes d'Armor, Plérin, France

f. Direction de la Recherche Clinique et de I'Innovation, Centre Oscar Lambret, Lille, France

g. Département d’Oncologie Médicale, Centre Antoine Lacassagne, Nice, France

h. Département d’Oncologie Médicale, Centre Francois Baclesse, Caen, France

i. Département d’Oncologie Médicale, Centre Paul Strauss, Strasbourg, France

j. Service d'Hépato-Gastroenterologie, Charles Nicolle University Hospital, Rouen, France

k. Institut de Cancérologie et d'Hématologie, Morvan University Hospital, Brest, France

I. Service d'Oncologie Digestive et d'Hépato-Gastroentrologie, La Timone University Hospital, Marseille, France 
m. Département d'Oncologie Médicale, Institut de Cancérologie de Lorraine, Université de Lorraine, Vandœuvre-lès-Nancy, France

n. Département d'Oncologie Médicale, Centre Georges-Francois Leclerc, Dijon, France

o. Service d'Hépato-Gastroenterologie, Francois Mitterrand University Hospital, Dijon

p. Univ. Lille, Department of Digestive and Oncological Surgery, Claude Huriez University Hospital, Lille, France

q. CESP, INSERM, Paris-Sud, Paris-Saclay University, 94805 Villejuif, France

Note: Parts of this study were previously presented at the 2016 ASCO Annual Meeting (oral presentation, Adenis A, et al. J Clin Oncol 2016;34:(Suppl)Abstract 4002) and at the 2016 World Gastro-Intestinal Congress (oral presentation, Bennouna J, et al. Ann Oncol 2016;27: (suppl_2),ii141.

Send correspondance to: Prof. Antoine Adenis, Département d’Oncologie Médicale, Institut Régional du Cancer de Montpellier, 208 rue des Apothicaires, 34298, Montpellier Cedex 5, France;

Telephone: +33 467614701; e-mail: antoine.adenis@icm.unicancer.fr

\# deceased 


\section{Abstract}

Purpose: The role of chemotherapy has not been established in the treatment of metastatic squamous-cell oesophageal cancer (mESCC).

Patients and methods: E-DIS is a discontinuation trial, aimed at estimating efficacy, quality of life and safety of chemotherapy continuation (CT-CONT) in mESCC patients who are free from progression after a selection-phase of chemotherapy. The primary endpoint was overall survival.

Results: 67 patients were randomised. The 9-month survival rate was 50\% (85\% $\mathrm{Cl}$ : $37-62 \%)$ and $48 \%(85 \% \mathrm{Cl}: 35-60 \%)$ in CT-CONT and in chemotherapy discontinuation (CT-DISC), respectively. The time until definitive deterioration of global health status (European Organisation for Research and Treatment of Cancer [EORTC] core quality of life questionnaire) was 6.6 months $(95 \% \mathrm{Cl}: 3.3-12.4)$ for $\mathrm{CT}-\mathrm{CONT}$, and 4.2 months $(95 \% \mathrm{Cl}$ :

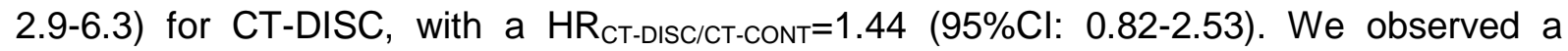
beneficial trend in favor of CT-CONT $(\mathrm{HR}>1)$ for most dimensions, including an improvement for three dimensions (dysphagia, eating, and oesophageal pain) of the EORTC Oesophageal Cancer Module QLQ-OES18.

Conclusion: CT-CONT provides an overall survival rate that is similar to CT-DISC. E-DIS provides valuable data to support shared decision-making between physicians and patients regarding CT- CONT/DISC.

Keywords: oesophageal cancer, chemotherapy, squamous-cell carcinoma, metastatic disease 


\section{Introduction}

Oesophageal cancer ranks eighth on the list of the most common cancers worldwide, with an estimated 456,000 new cases in 2012 [1]. Oesophageal squamous-cell carcinoma (ESCC) represents a majority of all cases of oesophageal cancer globally, and it is particularly prevalent in Eastern Asia, Eastern Africa and South America. ESCC is mainly related to the use of tobacco and alcohol [1]. Overall, this cancer is a deadly disease with a mortality-toincidence ratio of 0.88 [1]. Fifty per cent of these patients present with synchronous metastases at the time of diagnosis, and most patients who present initially with localised disease eventually develop metachronous metastases [2]. The 3-year survival rate for patients with metastatic oesophageal cancer is less than $1 \%$ [3].

The role of chemotherapy has not yet been fully established in the treatment of metastatic ESCC (mESCC) [4]. Many cytotoxic drugs promote tumor shrinkage [5-17] but, until now, there has been no randomised trial that provides unequivocal evidence for a clinical benefit with chemotherapy. For example, the European Organisation for Research and Treatment of Cancer (EORTC) reported the results of a randomised phase 2 trial that compared the activity of cisplatin (CDDP) with or without 5-fluorouracil (FU) in 88 mESCC [7]. Although a greater response rate was observed with the combination, this was achieved without any overall survival (OS) benefit, and at the expense of substantially increased toxicity, including deaths due to toxicity. Recently, the combination of chemotherapy and epidermal growth factor receptor monoclonal antibodies failed to improve the efficacy over chemotherapy alone $[18,19]$. Guidelines from the European Society of Medical Oncology (ESMO) recommend supportive care or chemotherapy as options in such settings [20]. Surprisingly, some physicians are convinced that mESCC patients may benefit from chemo. This attitude leads to the delivery of treatments with unproven benefit with side effects and unjustified costs.

The E-DIS trial (ClinicalTrials.gov identifier: NCT01248299) was a randomised discontinuation trial that was offered to mESCC patients who were free from progression after a 6-week selection-phase of FU/platinum-based chemotherapy. This study was 
designed to estimate the OS of mESCC patients who continued chemotherapy (CT-CONT arm).

\section{Patients and Methods}

\section{Patient Population and Study Design}

The multicenter E-DIS study selected patients before starting a first-line FU/platinum-based chemotherapy. Key selection criteria included histologically confirmed mESCC, measurable disease, age greater than 18 years, and an ECOG performance status of 0,1 or 2 . Prior chemotherapy was permitted only if it was delivered as a neoadjuvant treatment. The choice of the FU/platinum-based regimen was left physician's decision among the following ones: FU-CDDP, FU-CDDP-docetaxel, FU-folinic acid-CDDP (LVFU2-CDDP) and FU-folinic acidoxaliplatin combination (FOLFOX). Chemo dosing is detailed in Appendix Table S1 and in Table 1. Patients free from progression after 6 weeks of treatment were assigned equally to either continue the same chemotherapy or to discontinue chemotherapy. In the CT-CONT arm, the study treatment continued until there was disease progression, unacceptable toxicity, or a patient or physician decision to terminate the treatment. In the CT-DISC arm, chemotherapy could be resumed after disease progression. On-demand supportive care was offered to any patients. The protocol complied with the recommendations of the $18^{\text {th }}$ World Health Congress (Helsinki, Finland, 1964) and its subsequent amendments, good clinical practice guidelines, and other legal requirements. The protocol, including all amendments, was reviewed and approved by the CPP Nord-Ouest III ethics committee on August 22th, 2013. Patients provided written informed consent before enrollment in the study.

\section{Efficacy and Safety Assessments}

The primary end point was OS, defined as the time interval from the date of random assignment to the date of death from any cause. Secondary end points included progressionfree survival (PFS), QoL, safety and medical costs. Tumor assessment was performed 6 weeks and 12 weeks after the randomisation date and every 12 weeks thereafter. PFS was assessed according to RECIST 1.1. Quality of life (QoL) was assessed using the EORTC QoL core questionnaire (QLQ-C30) [21] and with the oesophagus-specific questionnaire 
(QLQ-OES-18) [22] at baseline and every 6 weeks thereafter until 42 weeks after randomisation. Adverse events (AE) were graded according to the National Cancer Institute Common Terminology Criteria version 4.0. Patients were observed until death or until 48 months after study entry.

\section{Statistical Considerations}

Sample size was calculated to ensure a precision of $12.5 \%$ to estimate the 9 -month OS rate with a two-sided $85 \%$ confidence interval $(85 \% \mathrm{Cl})$ in the CT-CONT arm. Assuming a 9month OS rate of 56\% [18], 31 patients were required in each arm. The CT-DISC arm served as an internal control. With an anticipated $70 \%$ eligibility rate for randomisation after 6 weeks of CT, 88 patients were required in the selection part of the trial in order to randomise 62 patients. In 2013, we found that the actual eligibility rate for the randomised part was only $58 \%$. The protocol was thus amended in order to select 106 patients. Randomisation was performed using a minimization method controlling for the following factors: previous chemotherapy (no vs yes), dysphagia (Atkinson grade 1-2 vs 3-4) and EQ-5D visual analog scale $(<40$ vs $\geq 40)$.

Statistical analyses of efficacy endpoints were performed per randomised arm by the intention-to-treat approach. Survival estimates were calculated per treatment arm using the Kaplan-Meier method from the date of random assignment. An unplanned and exploratory post hoc analysis was performed to estimate OS curves in CT-DISC patients according to whether or not they received post-progression chemotherapy. All randomised patients were included in the QoL analysis. For functional scales, QLQ-C30 and QLQ-OES-18 scores were considered as a definitive deterioration if the score decreased by more than 10 points compared with the score at randomisation, and without later improvement superior to 10 points compared with baseline. For symptom scales, a definitive deterioration was defined as an increase of 10 points or more without subsequent decrease. For each dimension of the QoL questionnaires, the time until definitive deterioration (TUDD) was defined as the time from randomisation to the first observation of a definitive deterioration of the corresponding score or death. Patients alive without reported definitive deterioration were censored at the 
date of the last follow-up visit. Patients without any QoL questionnaires were censored at randomization [23]. TUDD was estimated using the Kaplan-Meier method. The impact of treatments on the different dimensions of the QoL was estimated by hazard ratios $\left(\mathrm{HR}_{\mathrm{CT}}\right.$ DISC/CT-CONT) of QoL deterioration using Cox models. As QoL questionnaires could be missing during follow-up, leading to a possible overestimation of the TUDD, we also performed a sensitivity analysis considering the following imputations in the absence of a definitive deterioration of the QoL score: the date of definitive deterioration was imputed three weeks after the date of the last completion of the QoL questionnaire if the patient died more than two months later, or if the patient was alive without subsequent QoL; patients having completed all QoL questionnaires planned in the study were censored at the date of the last QoL questionnaire plus three weeks.

In the CT-CONT arm, treatment-related AEs were analysed considering the maximum grade per patient and per type of $A E$.

\section{Results}

Between January 2011 and February 2015, 105 patients were recruited in the selection phase of the study. The patient distribution in the trial is illustrated in Figure 1. Ninety patients were eligible for tumor assessment and 69 were found to be disease-controlled with an ECOG performance status of 0 to 2. Among them, 67 patients were randomly assigned, 34 to continue chemotherapy and 33 to discontinue chemotherapy. At the time of the final data extraction, the median follow-up for the randomised patients was 36.9 months.

Patient and treatment characteristics: Baseline characteristics and details of the treatments that were administered before and after randomisation are given in Appendix Table $\mathbf{S 1}$ and in Table 1 respectively. The two treatment groups were well balanced with regard to baseline characteristics of randomised patients, and of eligible and treated patients (data not shown). Among the 31 eligible and treated patients in the CT-CONT arm, 7 (23\%) received LVFU2CDDP and $24(77 \%)$ received FOLFOX. In the CT-DISC group, 24 patients resumed chemotherapy after having progressed, while 8 patients did not (missing data for 1 patient). 
Efficacy: At the time of the analysis, 53 deaths had been reported, 51 after disease progression, one due to hepatic encephalopathy 3.5 months, and one from an unknown cause 0.7 months, both in the CT-DISC arm. The estimated 9-month survival rate was $50 \%$ (85\% Cl: $37-62 \%)$ and $48 \%(85 \% \mathrm{Cl}: 35-60 \%)$ in the CT-CONT and the CT-DISC groups, respectively. The median OS rate was 8.5 months $(95 \% \mathrm{Cl}: 6.6$ to 12 months) and 8.8 months (95\% Cl: 5.9 to 13.4 months) for CT-CONT and CT-DISC, respectively. The median PFS was 4 months (95\% Cl: 2.8 to 5.8 months) and 1.4 months (95\% Cl: 1.4 to 2.7 months) for CT-CONT and CT-DISC, respectively. Kaplan-Meier survival curves are shown in Figure 2. As illustrated in Figure 3, the median OS was 9.9 months ( $95 \% \mathrm{Cl}: 6.3$ to 16.9 months) and 3.5 months $(95 \% \mathrm{Cl}: 2$ to 15.4 months) for the 24 patients $(24 / 33,72.7 \%)$ in the CTDISC group that received some post-progression chemotherapy and for the 8 patients that did not, respectively.

Quality of Life: QLQ-C30 and QLQ-OES18 domain scores showed no systematic differences between treatment arms at randomisation (data not shown). The median TUDD by treatment arm and Hazard Ratio (HR) for each dimension of both QoL questionnaires are displayed in Table 2 and Figure 4. We observed a trend for a benefit in favor of the CT-CONT arm $(H R>1)$ for most dimensions, with a significant difference for three dimensions of the QLQOES18: dysphagia with a median TUDD of 7.3 months (95\% Cl: 4.2-12.0) and 2.9 months (95\% Cl: 1.4-4.4) for CT-CONT and CT-DISC, respectively, leading to an estimated HR of $1.98(95 \% \mathrm{Cl}, 1.15-3.4), \mathrm{p}$-value=0.014; eating with a median TUDD of 7.7 months $(95 \% \mathrm{Cl}$ : 5.6-9.5) and 2.9 months (95\% Cl: 2.0-5.9), respectively, with $\mathrm{HR}=1.75$ (1.02-3.02), pvalue $=0.044$; and pain with a median TUDD of 8.1 months $(95 \% \mathrm{Cl}: 5.6-12.0)$ and 2.4 months (95\% Cl: 1.4-3.2), respectively, $\mathrm{HR}=2.52$ (1.43-4.43), $\mathrm{p}$-value=0.001. The results appeared stable in the sensitivity analysis (Appendix Table S3).

Adverse events and other assessments: There were no treatment-related deaths during the study. As detailed in Appendix Table S2, toxicity was mild and without unexpected AEs. The results of the medical cost analyses will be reported elsewhere. 


\section{Discussion}

Analysis of the primary end point for this multicenter, randomised, discontinuation phase-2 trial in mESCC patients who continued chemotherapy after having been disease-controlled with a FU/platinum regimen indicated a 50\% 9-month survival rate. Patients who had been assigned to CT-DISC had a $48 \% 9$-month survival rate. The median OS and median PFS were 8.5 and 4.0 months in the CT-CONT arm, respectively, and 8.8 and 1.4 months in the CT-DISC arm, respectively. Although one could have anticipated a better OS for patients in the CT-CONT arm that, actually, had been selected on the basis of early control of their disease, our survival results are of the same order of magnitude to those previously reported with FU/platinum regimens in contemporary randomised-studies [17-19]. E-DIS was not designed to detect a superiority of one arm over the other. Nevertheless, the two OS curves are so close and intertwined that one cannot claim a difference in OS. To understand why patients who discontinued chemotherapy had the same OS as patients who continued chemotherapy until progression, we examined the outcome of CT-DISC patients. First, most of these patients $(72.7 \%)$ received subsequent chemotherapy at progression. As a consequence, the majority of patients in the CT-DISC arm experienced a transient chemotherapy break rather than permanent discontinuation. Second, the shape of the OS curve for patients that resumed chemotherapy appeared better than the OS curve for patients that did not receive post-progression chemotherapy in the CT-DISC arm. Third, the OS curve for patients that resumed chemotherapy resembled those for patients that had been randomly allocated to the CT-CONT arm (Figure 3). Taken together, these features suggest that chemotherapy might have some favorable impact on the OS. However, we acknowledge that our observation is tentative, as the number of patients was low, and as we cannot rule out a selection bias, i.e., a poor performance status may have resulted in some patients not resuming chemotherapy.

In this study, most patients (77\%) received FOLFOX and $23 \%$ received LVFU2-CDDP. The latter, a biweekly regimen combining CDDP, bolus FU and infusional FU over 2 days, is regarded as a convenient way to deliver FU and CDDP and a safer regimen than the monthly 
FU-CDDP regimen with FU given as a continuous infusion over 4 or 5 days [24]. Oxaliplatin is a platinum derivative that has a more favorable toxicity profile than CDDP, and FOLFOX has been previously investigated in the treatment of mESCC with comparable efficacy, but with better safety than FU-CDDP [15]. Actually, FOLFOX is becoming popular in daily practice, since it has been shown to be equivalent to FU-CDDP in terms of efficacy in locally advanced settings $[25,26]$.

CT-CONT is associated with a significant delay in the worsening of some major symptoms such as dysphagia, eating and oesophageal pain (Figure 4). The results of the sensitivity analysis performed to control a possible overestimation of the time until definitive deterioration in the primary analysis of QoL were stable. Indeed, the study of the Qol has special relevance when life expectancy is short and when the benefit, if any, of some treatment can be perceived as modest. Consequently, it is satisfactory to observe an apparent benefit of the QoL in patients who continued chemotherapy.

Because of its limitations, E-DIS should be interpreted carefully. First, we acknowledge that part of our intervention was not standardised enough. At the onset of our trial, there was no clear evidence that chemotherapy provided a clinical benefit to mESCC patients, and ESMO guidelines suggested that either the best supportive care or palliative chemotherapy should be considered in this setting [20]. Consequently, we decided to accept all types of FU/platinum-based regimens as selection treatments. In 2009, at the time when the protocol was being written, we asked trial participants to provide on-demand supportive care to patients regardless of the treatment-arm to which they were assigned. Actually, we cannot certify that this supportive care was standardised adequately to avoid hypothetical differences between the two arms of this open-label trial, leading to a possible risk of systematically over-estimating the net clinical benefit of the control arm, as has been recently suggested [27], although we were referring to national recommendations that were supposed to be followed similarly in the two arms of this trial. Second, the main apparent benefit of CTCONT relies on QoL analysis, which was a secondary endpoint, with multiple comparisons 
and is subject to possible bias. Finally, E-DIS was designed as a non-comparative trial, therefore, all HRs are exploratory.

In conclusion, chemotherapy until progression provides an OS rate that is numerically similar to chemotherapy interruption in mESCC patients who had been disease-controlled with a 6week selection-course of a FU/platinum regimen. E-DIS provides valuable data to support shared decision-making between physicians and patients regarding CT-CONT/DISC.

Conflict of interest statement: None declared 


\section{References}

[1]. Ferlay J, Soerjomataram I, Dikshit R, Eser S, Mathers C, Rebelo M, et al. Cancer incidence and mortality worldwide: sources, methods and major patterns in GLOBOCAN 2012. Int J Cancer 2015;136:359-86.

[2]. Enzinger PC, Ilson DH, Kelsen DP. Chemotherapy in esophageal cancer. Semin Oncol 1999;5(Suppl 15):12-20.

[3]. Bouvier AM, Binquet C, Gagnaire A, Jouve JL, Faivre J, Bedenne L. Management and prognosis of esophageal cancers: has progress been made? Eur $\mathrm{J}$ Cancer 2006;42:228-33.

[4]. Adenis A, Penel N, Horn S, Dominguez S, Vanhuyse M, Mirabel X. Palliative chemotherapy does not improve survival in metastatic esophageal cancer. Oncology 2010;79: 46-54.

[5]. Ajani JA, Ilson DH, Daugherty K, Pazdur R, Lynch PM, Kelsen DP. Activity of taxol in patients with squamous cell carcinoma and adenocarcinoma of the esophagus. $J$ Natl Cancer Inst 1994;86:1086-9.

[6]. Conroy T, Etienne PL, Adenis A, Wagener DJ, Paillot B, François E, et al. Phase II trial of vinorelbine in metastatic squamous cell esophageal carcinoma. J Clin Oncol 1996;14:164-70.

[7]. Bleiberg H, Conroy T, Paillot B, Lacave AJ, Blijham G, Jacob JH, et al. Randomised phase II study of cisplatin and 5-fluorouracil (5-FU) versus cisplatin alone in advanced squamous cell oesophageal cancer. Eur J Cancer 1997;33:1216-20.

[8]. Ilson DH, Ajani J, Bhalla K, Forastiere A, Huang Y, Patel P, et al. Phase II trial of paclitaxel, fluorouracil, and cisplatin in patients with advanced carcinoma of the esophagus. J Clin Oncol 1998;16:1826-34.

[9]. Ilson DH, Saltz L, Enzinger P, Huang Y, Kornblith A, Gollub M, et al. Phase II trial of weekly irinotecan plus cisplatin in advanced esophageal cancer. J Clin Oncol 1999; 17:3270-5.

[10]. Conroy T, Etienne PL, Adenis A, Ducreux M, Paillot B, Oliveira J, et al. Vinorelbine and cisplatin in metastatic squamous cell carcinoma of the oesophagus: response, toxicity, quality of life and survival. Ann Oncol 2002;13:721-9.

[11]. Lordick F, von Schilling C, Bernhard H, Hennig M, Bredenkamp R, Peschel C. Phase II trial of irinotecan plus docetaxel in cisplatin-pretreated relapsed or refractory oesophageal cancer. Br J Cancer 2003;89:630-3.

[12]. Muro K, Hamaguchi T, Ohtsu A, Boku N, Chin K, Hyodo I, et al. A phase II study of single-agent docetaxel in patients with metastatic esophageal cancer. Ann Oncol 2004;15:955-9.

[13]. Burkart C, Bokemeyer C, Klump B, Pereira P, Teichmann R, Hartmann JT. A phase II trial of weekly irinotecan in cisplatin-refractory esophageal cancer. Anticancer Res 2007;27:2845-8.

[14]. Lee J, Im YH, Cho EY, Hong YS, Lee HR, Kim HS, et al. A phase II study of capecitabine and cisplatin (XP) as first-line chemotherapy in patients with advanced esophageal squamous cell carcinoma. Cancer Chemother Pharmacol 2008;62:7784.

[15]. Wang J, Chang J, Yu H, Wu X, Wang H, Li W, et al. A phase II study of oxaliplatin in combination with leucovorin and fluorouracil as first-line chemotherapy in patients with metastatic squamous cell carcinoma of esophagus. Cancer Chemother Pharmacol 2013;71:905-11. 
[16]. Lee SJ, Kim S, Kim M, Lee JY, Park YH, Im YH, et al. Capecitabine in combination with either cisplatin or weekly paclitaxel as a first-line treatment for metastatic esophageal squamous cell carcinoma: a randomized phase II study. BMC Cancer 2015;15:693.

[17]. Wang FH, Wang Y, Sun GP, Chen JH, Lin YC, Liu W, et al. Efficacy and safety of recombinant human lymphotoxin- $\alpha$ derivative with cisplatin and fluorouracil in patients with metastatic esophageal squamous cell carcinoma: A randomized, multicenter, open-label, controlled, phase $2 b$ trial. Cancer 2017;123:3986-94.

[18]. Lorenzen S, Schuster T, Porschen R, Al-Batran SE, Hofheinz R, Thuss-Patience P, et al. Cetuximab plus cisplatin-5-fluorouracil versus cisplatin-5-fluorouracil alone in first-line metastatic squamous cell carcinoma of the esophagus: a randomized phase II study of the Arbeitsgemeinschaft Internistische Onkologie. Ann Oncol 2009; 20:1667-73.

[19]. Moehler MH, Thuss-Patience PC, Brenner B, Longo F, Meiler J, Ettrich TJ, et al. Cisplatin/5-FU (CF) +/- panitumumab (P) for patients (pts) with non-resectable, advanced, or metastatic esophageal squamous cell cancer (ESCC): An open-label randomized AIO/TTD/BDGO/EORTC phase III trial (POWER). J Clin Oncol 2017; 35(suppl):abstr 4011.

[20]. Lordick F, Mariette M, Haustermans K, Obermannová R, Arnold D. Oesophageal cancer: ESMO Clinical Practice Guidelines for diagnosis, treatment and follow-up. Ann Oncol 2016; 27(Suppl 5):v50-v7.

[21]. Aaronson NK, Ahmedzai S, Bergman B, Bullinger M, Cull A, Duez NJ, et al. The European Organization for Research and Treatment of Cancer QLQ-C30: a qualityof-life instrument for use in international clinical trials in oncology. J Natl Cancer Inst 1993;85:365-76.

[22]. Blazeby JM, Conroy T, Hammerlid E, Fayers P, Sezer O, Koller M, et al. Clinical and psychometric validation of an EORTC questionnaire module, the EORTC QLQOES18, to assess quality of life in patients with oesophageal cancer. Eur J Cancer 2003;39:1384-94.

[23]. Anota A, Hamidou Z, Paget-Bailly S, Chibaudel B, Bascoul-Mollevi C, Auquier P, et al. Time to health-related quality of life score deterioration as a modality of longitudinal analysis for health-related quality of life studies in oncology: do we need RECIST for quality of life to achieve standardization? Qual Life Res 2015;24:5-18.

[24]. Bouché O, Raoul JL, Bonnetain F, Giovannini M, Etienne PL, Lledo G, et al. Randomized multicenter phase II trial of a biweekly regimen of fluorouracil and leucovorin (LV5FU2), LV5FU2 plus cisplatin, or LV5FU2 plus irinotecan in patients with previously untreated metastatic gastric cancer: a Federation Francophone de Cancerologie Digestive Group Study--FFCD 9803. J Clin Oncol 2004;22:4319-28.

[25]. Conroy T, Galais MP, Raoul JL, Bouché O, Gourgou-Bourgade S, Douillard JY, et al. Definitive chemoradiotherapy with FOLFOX versus fluorouracil and cisplatin in patients with oesophageal cancer (PRODIGE5/ACCORD17): final results of a randomised, phase 2/3 trial. Lancet Oncol 2014;15:305-14.

[26]. Bascoul-Mollevi C, Gourgou S, Galais MP, Raoul JL, Bouché O, Douillard JY, et al. Health-related quality of life results from the PRODIGE 5/ACCORD 17 randomised trial of FOLFOX versus fluorouracil-cisplatin regimen in oesophageal cancer. Eur $\mathrm{J}$ Cancer 2017;84:239-49. 
[27]. Nipp RD, Currow DC, Cherny NI, Strasser F, Abernethy AP, Zafar SY. Best supportive care in clinical trials: review of the inconsistency in control arm design. $\mathrm{Br}$ J Cancer 2015;113:6-11. 
The authors have declared no competing interest.

\section{Support and role of the Funding Source}

This work was supported by the French Ministry of Health (PHRC-K 2010 19-01). The funding source had no role in the design and conduct of the study; collection, management, analysis and interpretation of the data; preparation or approval of the manuscript and decision to submit the manuscript for publication

\section{Acknowledgments}

We are thankful for the contributions from patients, their families and other participating coinvestigators: C. Peugniez, N. Kotecki (Lille, France) and L. Bedenne (Dijon, France). We would also like to thank A. Kramar (Lille, France) for the fruitful discussion we had regarding the statistical plan, Aurélie Thenot-Maricourt (Lille, France) as Project Manager, and the Clinical Research Associates from our institution (Lille, France) for on-site data monitoring. The Northwest Data Center (CTD-CNO) is acknowledged for managing the data. This was supported by grants from the French National League Against Cancer (LNC) and the French National Cancer Institute (INCa). We also thank the UNICANCER Gastro-Intestinal group and for PRODIGE intergroup for their friendly support. Finally, we are deeply grateful to our colleague and friend, Professor Christophe Mariette (Univ. Lille, Department of Digestive and Oncological Surgery, Claude Huriez University Hospital, Lille, France) who passed away in July, 2017. Without his contribution and support this research project could not have been conducted. 


\section{TABLE and FIGURE LEGENDS}

Table 1 -Baseline characteristics of the 67 patients who were included in the randomised part of the study, by treatment group.

Table 2 - Quality of life analysis: median time until definitive deterioration (TUDD) by treatment group and Hazard Ratio for each dimension of quality of life questionnaires (QLQC30 and QLQ-OES18).

Figure 1 - CONSORT diagram. ITT, intention to treat

Figure 2 - Kaplan-Meier survival curves for (A) overall survival, and (B) progression-free survival according to treatment arm allocated by randomisation (intention-to-treat).

Figure 3 - Kaplan-Meier estimates of overall survival for patients who discontinued chemotherapy ( $n=33)$, according to whether $(n=24$, blue curve) or not ( $n=8$, yellow curve) they received post-progression chemotherapy. The dotted curve is the survival curve of patients ( $n=34)$ who were randomly assigned to continue chemotherapy.

Figure 4 - Relative treatment effect on the quality of life endpoints (Forest plot of the Hazard Ratio on definitive deterioration of quality of life)

Appendix Table S1 - Baseline characteristics of the 101 patients registered in the selection part of the study and who started chemotherapy, according to their subsequent participation in the randomised phase of the trial.

Appendix Table S2 - Maximal grade per patient and per type of adverse events related to the study treatment in CT-CONT arm.

Appendix Table S3 - Quality of life (sensitivity analysis): median time until definitive deterioration (TUDD) by treatment group and Hazard Ratio for each dimension in the quality of life questionnaires (QLQ-C30 and QLQ-OES18). 
Table 1 -Baseline characteristics of the 67 patients who were included in the randomised part of the study, by treatment group.

\begin{tabular}{|c|c|c|c|c|}
\hline & $\begin{array}{r}\mathrm{CT}- \\
\mathrm{N}\end{array}$ & $\begin{array}{l}\text { CONT } \\
=34\end{array}$ & & $\begin{array}{l}\text { DISC } \\
=33\end{array}$ \\
\hline Age in years, median (range) & 64.5 & $(43-81)$ & 63 & $(50-72)$ \\
\hline Gender & & & & \\
\hline Male & 25 & $74 \%$ & 29 & $88 \%$ \\
\hline Female & 9 & $26 \%$ & 4 & $12 \%$ \\
\hline ECOG performance status & & & & \\
\hline $0-1$ & 30 & $88 \%$ & 31 & $94 \%$ \\
\hline 2 & 4 & $12 \%$ & 2 & $6 \%$ \\
\hline No dysphagia & 16 & $50 \%$ & 17 & $52 \%$ \\
\hline Normal albumin & 22 & $65 \%$ & 21 & $66 \%$ \\
\hline Metachronous metastasis & 21 & $62 \%$ & 18 & $55 \%$ \\
\hline $\begin{array}{l}\text { Time interval between initial diagnosis and } \\
\text { first diagnosis of metastases, in months, median (range) }\end{array}$ & 10.9 & $(0-151)$ & 6.5 & $(0-41)$ \\
\hline Previous locoregional therapy & 20 & $59 \%$ & 19 & $58 \%$ \\
\hline Number of metastatic sites & & & & \\
\hline $\begin{array}{l}>1 \\
\text { Main metastatic sites }{ }^{(1)}\end{array}$ & 22 & $65 \%$ & 14 & $42 \%$ \\
\hline Lung & 16 & $47 \%$ & 20 & $61 \%$ \\
\hline Liver & 15 & $44 \%$ & 10 & $30 \%$ \\
\hline Nodes & 25 & $74 \%$ & 19 & $58 \%$ \\
\hline Bones & 3 & $9 \%$ & 0 & $0 \%$ \\
\hline Others & 8 & $24 \%$ & 6 & $18 \%$ \\
\hline Previous chemotherapy in the neoadjuvant setting & 18 & $53 \%$ & 19 & $58 \%$ \\
\hline Chemotherapy regimen & & & & \\
\hline FU-CDDP ${ }^{(2)}$ & $0 / 34$ & $0 \%$ & $1 / 33$ & $3 \%$ \\
\hline FU-CDDP-docetaxel ${ }^{(3)}$ & $0 / 34$ & $0 \%$ & $3 / 33$ & $9 \%$ \\
\hline LVFU2-CDDP $^{(4)}$ & $8 / 34$ & $24 \%$ & $4 / 33$ & $12 \%$ \\
\hline FOLFOX ${ }^{(5)}$ & $26 / 34$ & $76 \%$ & $25 / 33$ & $76 \%$ \\
\hline Response after 6 weeks of chemotherapy & & & & \\
\hline Complete response & 0 & & 1 & $3 \%$ \\
\hline Partial response & 12 & $35 \%$ & 9 & $27 \%$ \\
\hline Stable disease & 20 & $59 \%$ & 21 & $64 \%$ \\
\hline Progressive disease & 2 & $6 \%$ & 0 & \\
\hline Missing data & & & $2^{(6)}$ & $6 \%$ \\
\hline
\end{tabular}

(1) One patient can have several metastatic sites.

(2) FU-CDDP: FU $1,000 \mathrm{mg} / \mathrm{m}^{2}$ as a continuous infusion over $96 \mathrm{~h}$, plus CDDP $100 \mathrm{mg} / \mathrm{m}^{2}$ day 1 or 2 , every 4 weeks.

(3) FU-CDDP-docetaxel: docetaxel $75 \mathrm{mg} / \mathrm{m}^{2}$ on day 1 , CDDP $75 \mathrm{mg} / \mathrm{m}^{2}$ on day 1 , and FU $750 \mathrm{mg} / \mathrm{m}^{2}$ as a continuous infusion over $120 \mathrm{~h}$, every 4 weeks.

(4) LVFU2-CDDP: FU-folinic acid-CDDP, with CDDP $50 \mathrm{mg} / \mathrm{m}^{2}$, folinic acid $200 \mathrm{mg} / \mathrm{m}^{2}$, and bolus FU $400 \mathrm{mg} / \mathrm{m}^{2}$ on day 1 followed by FU 2,400 mg/m² as a continuous infusion over $46 \mathrm{~h}$, every 2 weeks.

(5) FOLFOX: FU-folinic acid-oxaliplatin combination with oxaliplatin $85 \mathrm{mg} / \mathrm{m}^{2}$, folinic acid $200 \mathrm{mg} / \mathrm{m}^{2}$, and bolus

FU $400 \mathrm{mg} / \mathrm{m}^{2}$ on day 1 followed by FU $2,400 \mathrm{mg} / \mathrm{m}^{2}$ as a continuous infusion over $46 \mathrm{~h}$, every 2 weeks.

(6) For 2 patients, known as non-progressive at 6 weeks, detailed information about the tumour response at 6 weeks is not available. 
Table 2 - Quality of life analysis: median time until definitive deterioration (TUDD) by treatment group and Hazard Ratio for each dimension of the quality of life questionnaires (QLQ-C30 and QLQ-OES18)

\begin{tabular}{|c|c|c|c|}
\hline Dimension & $\begin{array}{l}\text { Median TUDD i } \\
\text { CI) } \\
\text { CT-CONT arm }\end{array}$ & $\begin{array}{l}\text { months (95\% } \\
\text { CT-DISC arm }\end{array}$ & $\begin{array}{c}\text { HR }_{\text {CT-DISC } / \text { CT-CONT }}(95 \% \\
\text { Cl) }\end{array}$ \\
\hline \multicolumn{4}{|l|}{ QLQ-C30 questionnaire } \\
\hline Global health status & $6.6(3.3-12.4)$ & $4.2(2.9-6.3)$ & $1.44(0.82-2.53)$ \\
\hline Physical & $7.3(5.6-9.4)$ & $5.4(2.9-8.5)$ & $0.96(0.56-1.64)$ \\
\hline Role & $5.6(3.1-8.1)$ & $4.5(3.2-8.3)$ & $1.01(0.59-1.72)$ \\
\hline Emotional & $7.1(4.2-11.9)$ & $5.6(3.2-7.8)$ & $1.38(0.80-2.37)$ \\
\hline Cognitive & $7.8(3.3-12)$ & $4.1(2.8-8.5)$ & $1.40(0.80-2.42)$ \\
\hline Social & $5.6(3.0-8.1)$ & $6.2(4.2-8.8)$ & $0.88(0.51-1.53)$ \\
\hline Fatigue & $5.6(2.8-9.6)$ & $4.4(2.9-6.4)$ & $1.28(0.73-2.22)$ \\
\hline Nausea & $7.8(3.3-9.8)$ & $5.4(2.8-8.8)$ & $1.30(0.75-2.24)$ \\
\hline Pain & $5.6(2.8-7.0)$ & $2.9(2.1-6.3)$ & $1.09(0.64-1.86)$ \\
\hline Dyspnea & $7.3(4.2-11.9)$ & $4.4(2.8-7.8)$ & $1.45(0.84-2.50)$ \\
\hline Insomnia & $7.3(3.3-11.9)$ & $5.4(2.0-7.8)$ & $1.46(0.84-2.55)$ \\
\hline Appetite loss & $7.1(5.2-12.0)$ & $4.5(2.9-7.8)$ & $1.45(0.82-2.58)$ \\
\hline Constipation & $7.3(5.2-11.9)$ & $5.7(2.8-9.9)$ & $1.26(0.73-2.18)$ \\
\hline Diarrhea & $6.6(3.3-9.9)$ & $4.5(2.9-8.5)$ & $1.23(0.70-2.16)$ \\
\hline Financial difficulties & $8.1(5.6-12.4)$ & $6.3(3.2-9.9)$ & $1.30(0.74-2.27)$ \\
\hline Pain alone & $5.6(3.0-7.3)$ & $5.4(2.8-8.3)$ & $1.03(0.60-1.77)$ \\
\hline \multicolumn{4}{|l|}{$\begin{array}{l}\text { QLQ-OES18 } \\
\text { questionnaire }\end{array}$} \\
\hline Dysphagia & $7.3(4.2-12)$ & $2.9(1.4-4.4)$ & $1.98(1.15-3.40)$ \\
\hline Eating & $7.7(5.6-9.5)$ & $2.9(2.0-5.9)$ & $1.75(1.02-3.02)$ \\
\hline Reflux & $7.8(4.7-11.9)$ & $3.2(1.4-7.8)$ & $1.63(0.93-2.85)$ \\
\hline Pain & $8.1(5.6-12.0)$ & $2.4(1.4-3.2)$ & $2.52(1.43-4.43)$ \\
\hline Trouble swallowing & $7.8(5.2-11.9)$ & $6.3(3.2-9.1)$ & $1.39(0.81-2.41)$ \\
\hline Choked when swallowing & $7.1(4.2-9.9)$ & $5.4(3.0-6.9)$ & $1.29(0.75-2.22)$ \\
\hline Dry mouth & $6.8(3.3-9.8)$ & $5.9(3.1-9.9)$ & $0.98(0.57-1.67)$ \\
\hline Taste & $7.3(5.2-9.5)$ & $4.4(2.9-7.8)$ & $1.19(0.70-2.03)$ \\
\hline Coughing & $8.1(5.6-9.9)$ & $6.3(3.2-8.8)$ & $1.17(0.69-1.98)$ \\
\hline Talking & $7.3(3.3-9.5)$ & $5.7(3.2-8.3)$ & $0.98(0.58-1.66)$ \\
\hline
\end{tabular}




\section{Figure legends}

Figure 1 - CONSORT diagram. ITT, intention to treat

Figure 2 - Kaplan-Meier survival curves for (A) overall survival, and (B) progression-free survival according to treatment arm allocated by randomisation (intention-to-treat).

Figure 3 -Kaplan-Meier estimates of overall survival for patients who were randomly assigned to discontinue chemotherapy (CT-DISC, $n=33)$, according to whether $(n=24$, blue curve) or not ( $n=8$, yellow curve) they received post-progression chemotherapy (PPC). The dotted curve is the survival curve of patients $(n=34)$ who were randomly assigned to continue chemotherapy (CT-CONT).

Figure 4 - Relative treatment effect on the quality of life endpoints (Forest plot of the Hazard Ratio on definitive deterioration of quality of life).

The HR of definitive deterioration in the CT-DISC group compared to the CT-CONT group. The 95\% confidence intervals are estimated in a Cox model for each component separately. 


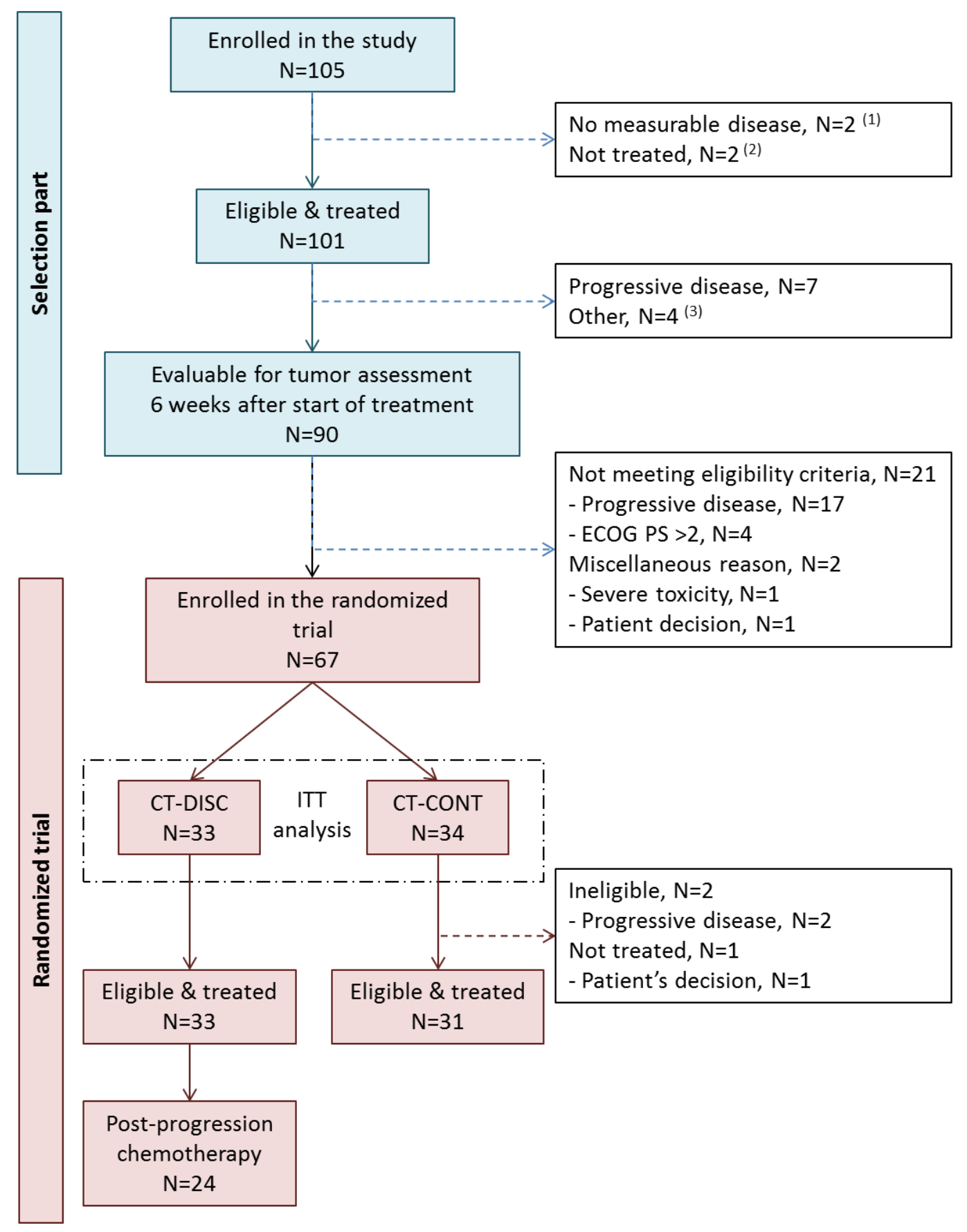

(1) Two patients with an adenocarcinoma were withdrawn from the study after enrolment because they were classified as non-eligible (no measurable disease at study entry).

(2) Two patients were not treated due to poor general health status.

(3) Four patients had no tumor evaluation 6 weeks after start of treatment, due to patient's decision, no compliance, concurrent disease, and poor performance status. 
A

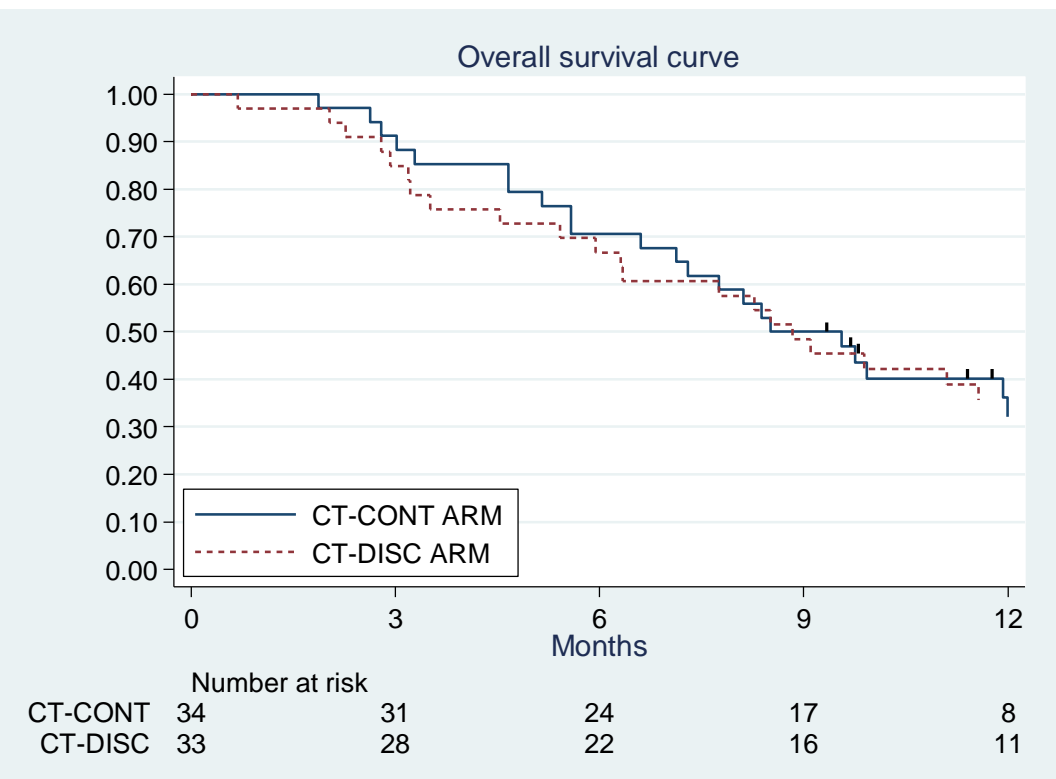

B

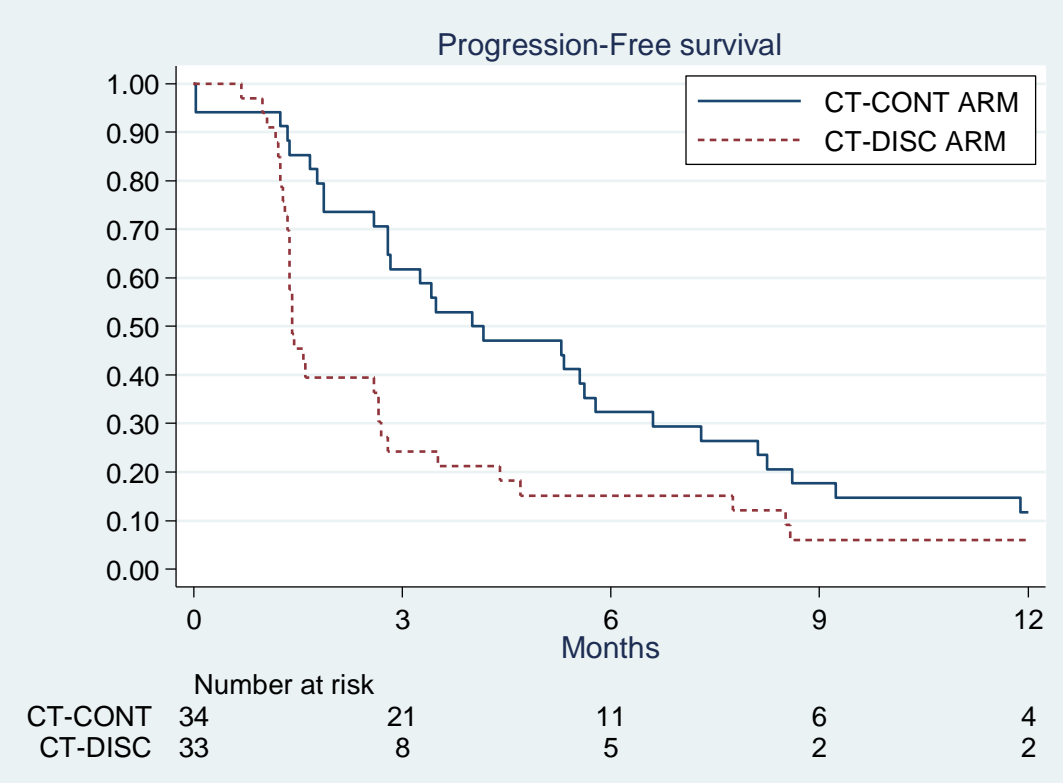




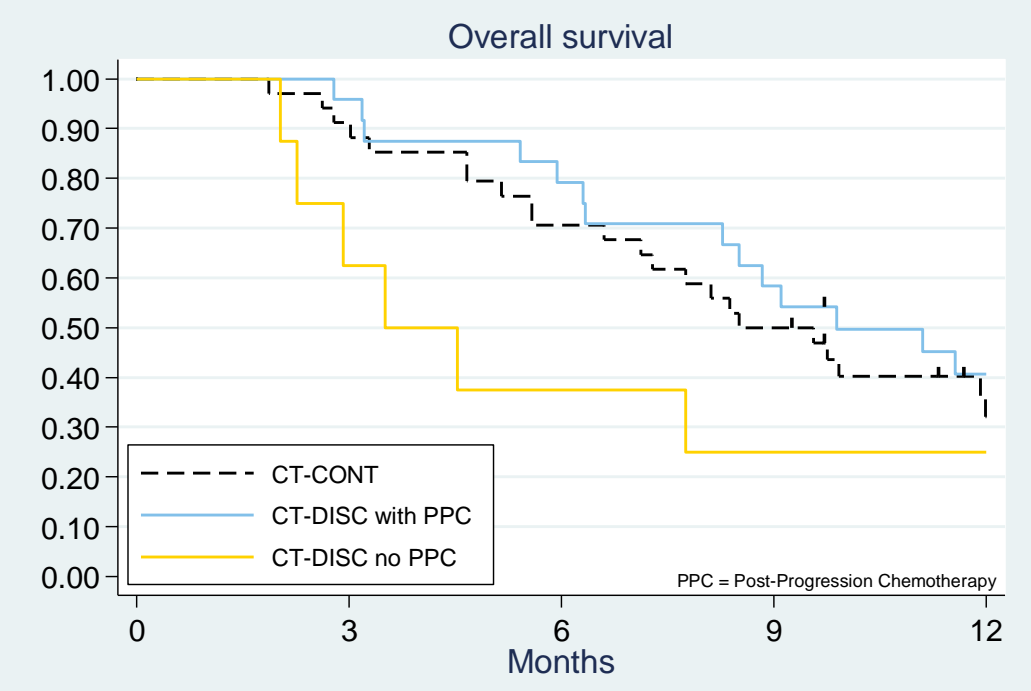

CT-CONT Number at risk 


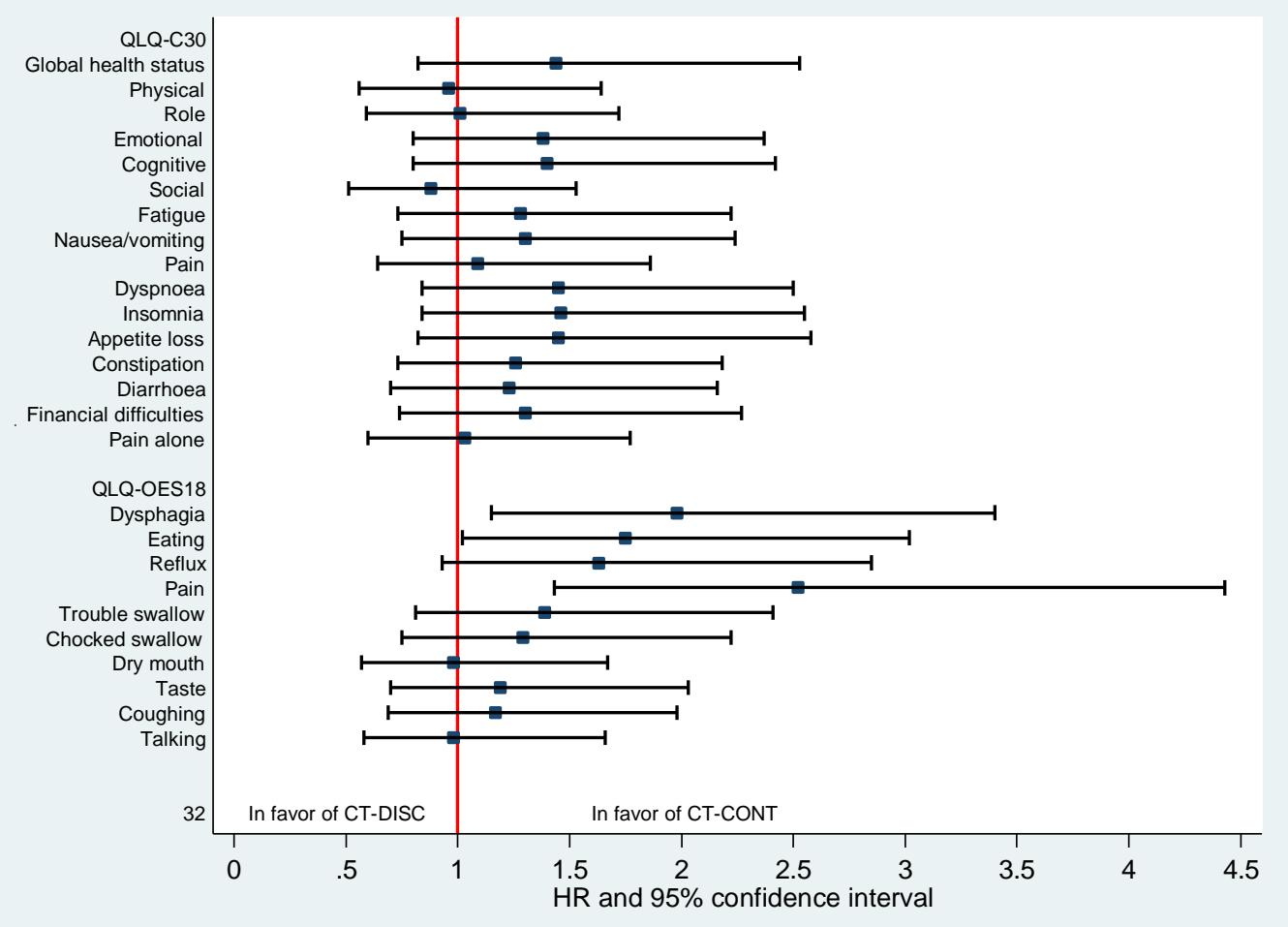

